\title{
OPTICAL IDENTIFICATION OF ROSAT ALL-SKY SURVEY GALAXY CLUSTER CANDIDATES
}

\author{
B.J. MCLEAN ${ }^{1}$, H. BÖHRINGER ${ }^{2}$, R. BURG ${ }^{1,3}$, R. GIACCONI ${ }^{1,3,4}$, J.P. HUCHRA $^{5}$, \\ W. VOGES ${ }^{2}$ \\ ${ }^{1}$ Space Telescope Science Institute, 3700 San Martin Drive, Baltimore, MD 21218, \\ U.S.A. \\ ${ }^{2}$ Max-Planck Intitut für Extraterrestrische Physik, Karl-Schwarzschild-Strasse 2, \\ W-8046 Garching, Germany \\ ${ }^{3}$ Johns Hopkins University, Baltimore, MD 21218, U.S.A. \\ ${ }^{4}$ European Southern Observatory, Karl-Schwarzschild-Strasse 2, W-8046 Garching, \\ Germany \\ ${ }^{5}$ Smithsonian Astrophysical Observatory, Cambridge, MA 02138, U.S.A.
}

\section{Introduction}

Clusters of galaxies are used to study large scale structure and evolution in the universe. The luminosity functions of clusters can be used to investigate their evolutionary effects while their spatial and angular correlation functions are a measure of large-scale clustering. There are, however, many problems with the traditional cluster catalogues that were derived by visual inspection of plate material. The widely used Abell catalogue (Abell 1958) has been shown to have significant problems in homogeneity and completeness (Postman et al. 1986).

Automated measuring machines to scan Schmidt plates of large areas of the sky and to produce objective catalogues are beginning to enable substantial progress in providing homogeneous, statistically complete samples. The first of these to be published is the Edinburgh/Durham Cluster Catalogue (Lumsden et al. 1992), based on the COSMOS data for 60 Schmidt plates from the UKSTU centered on the SGP. A similar effort is underway at STScI to produce a galaxy catalogue from the POSS-E plates for the northern hemisphere. Although these catalogues provide objective measurements and rigorous selection criteria, they still suffer from chance alignments which may affect both cluster richness measures and produce spurious clusters.

The ROSAT X-ray all-sky survey has been used to create a catalogue of cluster candidates using the X-ray properties only. Since $\mathrm{X}$-ray emission is proportional to the square of the gas density, clusters have the signature of being extended in X-ray maps. The presence of the X-ray emission is indicative of an intracluster medium and implies a bound system that is, or in the process of being virialised, eliminating chance alignments.

An international team (MPE, CfA, STSCI/JHU) has been formed to create a new catalogue of northern clusters. The first step is the identification of extended X-ray sources (candidate clusters) above a given flux limit using the ROSAT standard analysis software system as applied to the all-sky survey data. These candidates are then matched to the STScI plate catalogues to check on their optical properties, to eliminate very large, nearby galaxies and bright stars which 
can contaminate the sample. Additional CCD observations and redshift measurements are being carried out for the sample.

\section{Plate Processing}

The Guide Star Selection System (GSSS) group has built up an image archive of all the Schmidt plates that were scanned with the measuring machines at STScI. Over 2500 plates digitised at a resolution of 1.7 arcsecond per pixel have been stored on WORM optical discs and are routinely used to support HST operations and staff research. We are using the POSS-I E plates from the archive to support identification of ROSAT sources (McLean \& Burg 1992). In the future, we plan to use the better POSS-II plate material that is being scanned at a higher resolution.

Each of the digitised plates are processed to obtain an object catalogue with positions, magnitudes and classifications. One of the major problems is that the POSS-I plates do not have sensitometer spots or calibration wedges superimposed upon them. For this reason, we have modified the areal profile technique used by the APM group (Bunclark 1982) to determine the stellar profile and D-I curve for each plate. The GSPC (Guide Star Photometric Catalogue, Lasker 1988) and available observations from the GSPC-II (Postman et al. 1992) are being used as standards for each plate. These catalogues provide preliminary information on the cluster candidates (see plate 1). An analysis of these data is underway and will be presented at a later date.

\section{Cluster Candidates}

A preliminary catalogue of cluster candidates was derived from the ROSAT sky survey observations (Giacconi \& Burg 1992). The selection criteria used were a flux limit of $2 \times 10^{-12}$ erg $\mathrm{sec}^{-1} \mathrm{~cm}^{-2}$, a minimum X-ray diameter of 1 arcminute, $\delta>0^{\circ}$ and $\left|\mathrm{b}^{\mathrm{II}}\right|>20^{\circ}$.

This initial catalogue used a bright flux limit as it is based on the preliminary ROSAT calibrations. Later versions may lower this flux limit to detect lower luminosity and more distant clusters. These criteria have provided us with 522 candidates in a flux and diameter limited sample. The objects were then correlated with the Guide Star Catalogue and the STScI POSS E catalogues to check their optical morphology, with the Abell catalogue to check for known clusters, and with the CFA ZCAT for previously measured redshifts. The current categorisation is as follows: 191 Abell clusters, 222 other clusters/groups, 17 nearby galaxies, 32 stars, 16 active galaxies (QSO/Seyfert/BL Lac) and 44 unidentified objects in blank fields.

The $1.2 \mathrm{~m}$ telescope at Mt. Hopkins Observatory is being used to obtain $\mathrm{R}$ band images of the cluster candidates. These images provide the finding charts for the MMT observation for those clusters that are too faint to be measured reliably from the POSS-E plates, and are also being used to provide data for determining luminosity functions and morphological studies of the clusters, particularly looking for differences between the Abell clusters and the newly detected ones.

The MMT multi-object spectrograph is being used to obtain redshifts of the cluster candidates that do not have previously measured redshifts with the emphasis on obtaining data for all clusters in the range of 9-14 hours Right Ascension to provide a complete subsample for analysis. These observations are being reduced. 

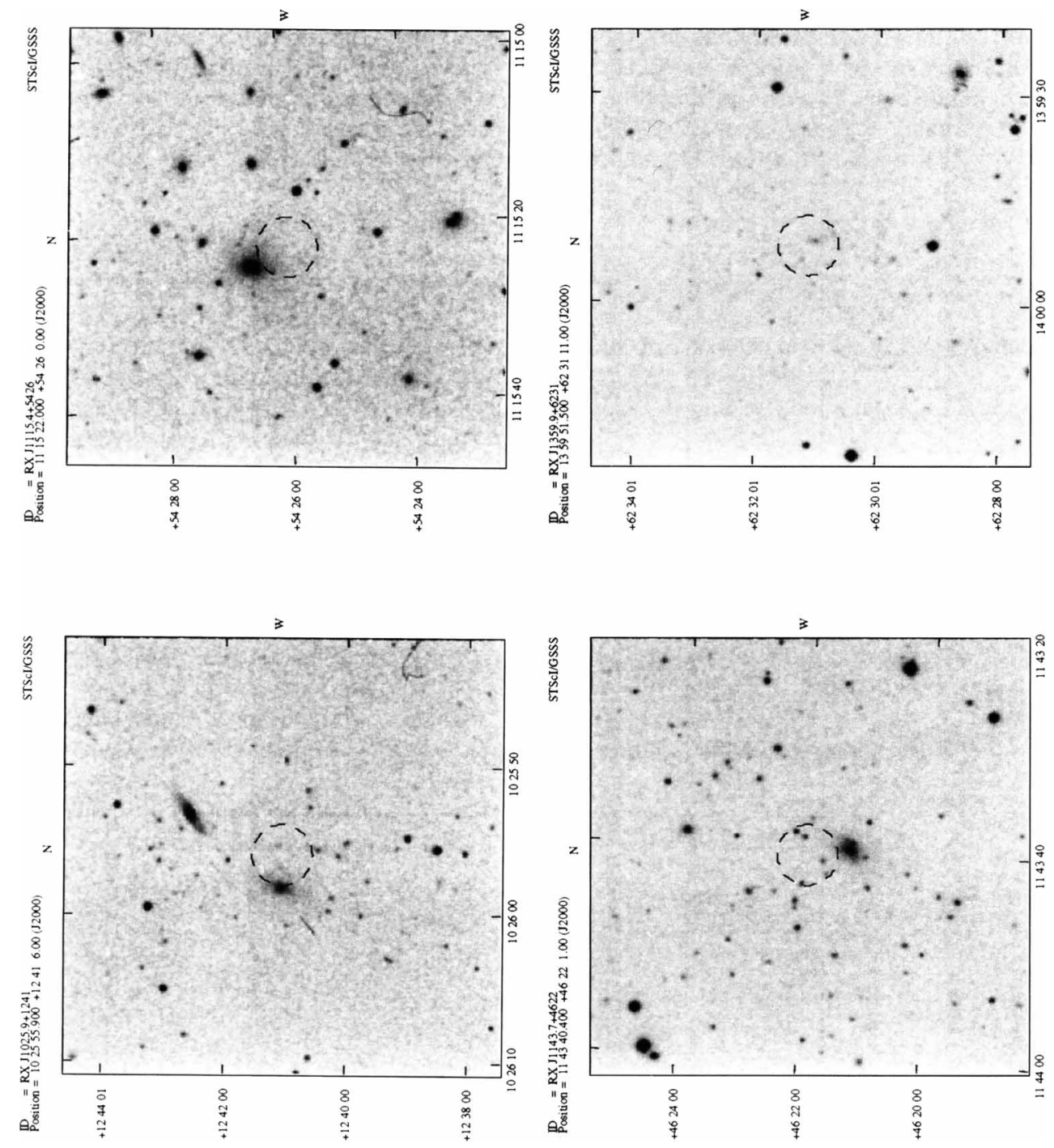

Plate 1. Examples of X-ray source fields taken from the STScI image archive that are identified with clusters. $\mathrm{RXJ1025} .9+1241$ at redshift $=0.14 ; \mathrm{RXJ1115.4+5426}$ at redshift $=0.07 ; \mathrm{RXJ1143.7+4622}$ (Abell 1361 ) at redshift $=0.12 ; \mathrm{RXJ} 1359+6231$ at redshift $=0.32$. 


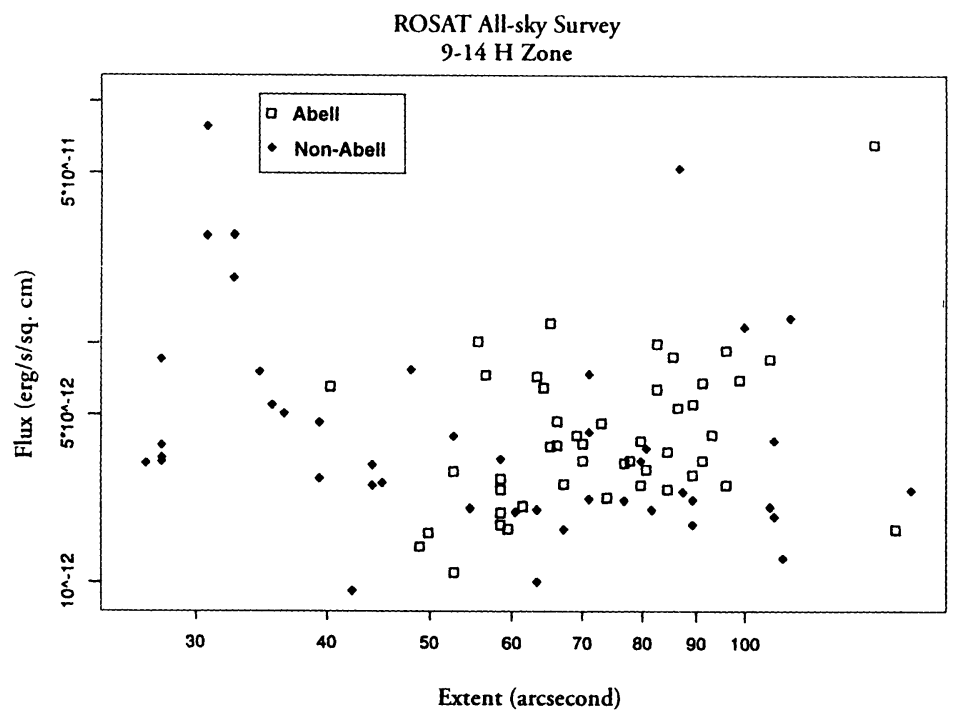

Figure 1. X-ray extent v. Flux.

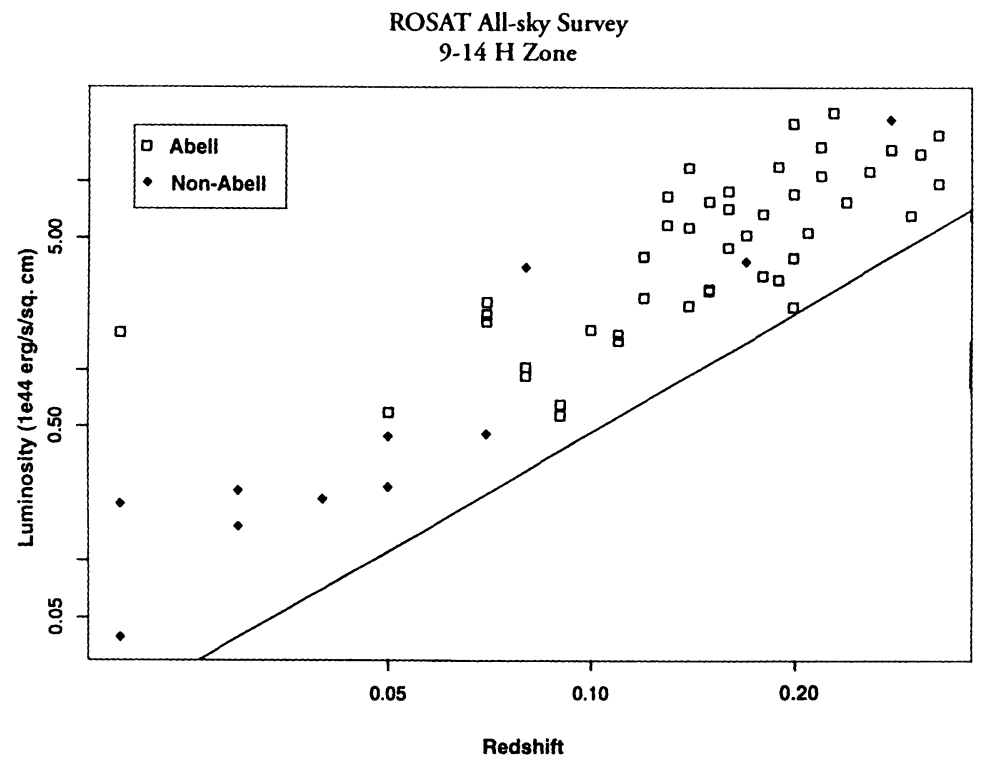

Figure 2. Redshift v. X-ray luminosity. 
In the case of apparently blank fields, the deeper CCD images (typical exposures are $2 \times 15$ minutes in the $R$ band) revealed faint clusters for about half of the candidates, with the MMT spectroscopy confirming that the remaining half are AGN. It is to be noted in Figs 1 \& 2 , however, that the luminosity and distance of clusters, not identified by Abell but detected in our sample, extend to the full range of luminosity and distances spanned by the Abell clusters up to luminosities of $>10^{15}$ ergs $\sec ^{-1}$ and distances corresponding to a redshift of about 0.45 .

\section{References}

Abell, G.O., 1958. Astrophys. J. Suppl., 3, 211.

Bunclark, P.S., 1982. 'Proceedings of the Workshop on Astronomical Measuring Machines (Edinburgh)', eds. Stobie \& McInnes.

Giacconi, R. and Burg, R., 1993. 'Proceedings of Milan Cosmology Congress', eds. Chincarini and Maccacaro, in press.

Lucey, J.R., 1983. Mon. Not. R. astron. Soc., 294, 33.

Lumsden, S.L., Nichol, R.C., Collins, C.A. and Guzzo, L., 1992. Mon. Not. R. astron Soc., 258, 1.

McLean, B.J. and Burg, R., 1992. In 'Digitised Optical Sky Surveys', eds. H.T. MacGillivray and E.B. Thomson, Kluwer Academic Publishers, p. 465.

Postman, M., Geller, M.J. and Huchra, J.P., 1986. Astron. J., 91, 1267.

Postman, M., Siciliano, L., Shara, M., Rehner, D., Brosch, N., Sturch, C, Bucciarelli, B. and Lopez, C., 1992. In 'Digitised Optical Sky Surveys', eds. H.T. MacGillivray and E.B. Thomson, Kluwer Academic Publishers, p. 6.

Scaramella, R., Zamorani, G., Vettolani, G. and Chincarini, G., 1991. Astron. J., 101, 342. 